\title{
Itinerant ferromagnetism in a two-dimensional atomic gas
}

\author{
G.J. Conduit ${ }^{1,2, \text { * }}$ \\ ${ }^{1}$ Department of Condensed Matter Physics, Weizmann Institute of Science, Rehovot 76100, Israel \\ ${ }^{2}$ Physics Department, Ben Gurion University, Beer Sheva 84105, Israel
}

(Dated: June 20, 2018)

\begin{abstract}
Motivated by the first experimental evidence of ferromagnetic behavior in a three-dimensional ultracold atomic gas, we explore the possibility of itinerant ferromagnetism in a trapped twodimensional atomic gas. Firstly, we develop a formalism that demonstrates how quantum fluctuations drive the ferromagnetic reconstruction first order, and consider the consequences of an imposed population imbalance. Secondly, we adapt this formalism to elucidate the key experimental signatures of ferromagnetism in a realistic trapped geometry.

PACS numbers: 03.75.Ss, 75.20.En, 64.60.Kw, 75.45. $+\mathrm{j}$
\end{abstract}

\section{INTRODUCTION}

Itinerant ferromagnetism is a ubiquitous strongly correlated phase of matter in the solid state. The theoretical study of itinerant ferromagnetism dates back to the pioneering work of Stoner [1] and Wohlfarth, which showed that ferromagnetism emerges as repulsive pairwise interactions between electrons overcome the kinetic energy penalty of polarization. Subsequent theoretical work has determined that soft transverse magnetic fluctuations have the potential to drive the ferromagnetic transition first order before the quantum critical point is reached [3 [8]. Phenomena consistent with a first order transition have been observed in the solid state; though it is difficult to determine whether they are due to soft magnetic fluctuations or the coupling of the magnetic moment to phonon degrees of freedom. However, Jo et al. 9 have recently presented the first tentative evidence [10, 11] of itinerant ferromagnetism in an ultracold atomic gas. The cold atom gas is a clean system in which to study ferromagnetism, completely devoid of the interfering phonon degrees of freedom encountered in the solid state, so gifts investigators with a valuable tool with which to answer long-standing questions about solid state ferromagnets. Furthermore, ultracold atoms experiments also present a unique opportunity to explore fundamentally new physics associated with ferromagnetism including the consequences of population imbalance [7], a conserved net magnetization [12], the damping of fluctuations by three-body loss [13], spin drag [14], and mass imbalance. Here we aim to take advantage of the high levels of control investigators can exercise over the external potential trapping the gas and turn to study ferromagnetism in a two-dimensional thin film.

Itinerant ferromagnetism is difficult to observe in two dimensions in the solid state [15, 16]. However, it could be realized in an ultracold atom gas by using counterpropagating lasers to create one-dimensional potential

*Electronic address: gjc29@cam.ac.uk which will lead to a stacked two-dimensional gas. The system also offers investigators the opportunity to study the possibility for a superconducting instability to emerge near to the ferromagnetic phase transition [17]. The twodimensional system is of particular interest in this case as it could shed light on high temperature superconductivity where antiferromagnetism competes with d-wave superconductivity to form the ground state. Here we adapt the formalism introduced for the three-dimensional case [7] to expose the contrasting behavior of the two-dimensional ferromagnet. We develop a formalism that captures the effects of transverse quantum fluctuations and explore how they renormalize the effective interaction strength. We then address how population imbalance modifies the behavior of the atomic gas before studying ferromagnetic ordering in a trapped geometry.

\section{FIELD INTEGRAL FORMALISM}

It has been long established that quantum fluctuations in a three-dimensional fermionic gas with repulsive interactions have the potential to drive the ferromagnetic transition first order [4 [8]. To investigate the impact of quantum fluctuations in a two-dimensional fermionic gas we explore ferromagnetic reconstruction within the setting of an atomic gas, adapting the phenomenology developed for the three-dimensional case in Ref. [7]. We adopt this formalism because unlike the Eliashberg theory [3] it provides an exact expression for the free energy which then allows us to make a prediction of the critical interaction strength for the onset of ferromagnetism and study the atomic gas within a harmonic well. Moreover, ab initio Quantum Monte Carlo calculations [8, 18] have recently been used to verify the three-dimensional formalism, which should therefore provide a solid foundation from which to study the two-dimensional case. Although the atoms do not carry spin, we discriminate between the two fermionic species with a pseudospin $\sigma \in\{\uparrow, \downarrow\}$. The species cannot interconvert so separate chemical potentials $\mu_{\sigma}$ tune the population imbalance, which in turn pins the net polarization along the pseudospin direction. However, when the spontaneous mag- 
netization formed exceeds the population imbalance, a nonzero in-plane magnetization emerges. To study the potential for ferromagnetic ordering we express the partition function as a fermionic coherent state path integral $\mathcal{Z}=\operatorname{Tr} \mathrm{e}^{-\beta(\hat{H}-\mu \hat{N})}=\int \mathcal{D} \psi \mathrm{e}^{-S}$ with the action

$$
S=\int \sum_{\sigma=\{\uparrow, \downarrow\}} \bar{\psi}_{\sigma}\left(\partial_{\tau}+\epsilon_{\hat{\mathbf{k}}}-\mu_{\sigma}\right) \psi_{\sigma}+\int g \bar{\psi}_{\uparrow} \bar{\psi}_{\downarrow} \psi_{\downarrow} \psi_{\uparrow}
$$

Here $\int \equiv \int_{0}^{\beta} \mathrm{d} \tau \int \mathrm{d}^{2} r$ with reduced temperature $\beta=$ $1 / k_{\mathrm{B}} T$, and $\epsilon_{\hat{\mathbf{k}}}$ denotes the dispersion. As we wish to investigate two-dimensional ferromagnetism we have constrained the spatial integral to a plane. A twodimensional atomic gas could be realized experimentally using counter-propagating laser beams whose antinodes at half-wavelength spacing $b$ will define stacked quasitwo-dimensional layers. Though at finite temperature the ferromagnetic ordering is only marginally stable, longrange order should be stabilized by the weak inter-plane coupling [12]. The repulsive contact interaction parameter $g=g \delta^{3}(\mathbf{r})$ that can be tuned with a Feshbach resonance [2] is linked to the s-wave scattering length $a$ in three dimensions through $g=\sqrt{2 / \pi} a / b[23]$. Unique to two dimensions, the interaction strength is independent of density. This means that within a trapped geometry the entire atomic gas will experience the same effective interaction strength and therefore adopt the same polarization.

To develop an effective Landau theory of the magnetic transition, Hertz introduced a scalar HubbardStratonovich decoupling of the two-body interaction term in the spin channel [2]. However, this form of decoupling neglects the potential impact of soft transverse field fluctuations, which in three dimensions are responsible for driving the second order transition first order [7, 8]. Therefore, we will introduce a general Hubbard-Stratonovich decoupling that incorporates fluctuations in all of the spin $\phi$ and charge $\rho$ sectors. Integrating over the fermion degrees of freedom yields $\mathcal{Z}=\int \mathrm{e}^{-S} \mathcal{D} \phi \mathcal{D} \rho$ with the action

$$
S=\int g\left(\phi^{2}-\rho^{2}\right)-\operatorname{Tr} \ln \left[\partial_{\tau}+\epsilon_{\hat{\mathbf{k}}}-\mu_{\sigma_{z}}+g \rho-g \boldsymbol{\sigma} \cdot \boldsymbol{\phi}\right] .
$$

At this stage a saddle point analysis would determine the mean-field values of $\rho$ and $\phi$. However, quadratic fluctuations in these auxiliary fields renormalize these equations. Therefore we introduce the putative saddle point values $\rho_{0}$ for density and $\mathbf{m}$ for magnetization, integrate out fluctuations in the auxiliary fields, and finally minimize the energy to determine $\rho_{0}$ and $\mathbf{m}$. It is also convenient to rotate the z-axis from the quantization axis to lie along the direction of the saddle point magnetization $\mathbf{m}$, with components labeled by $s \in\{+,-\}$. After integrating over fluctuations in both the density $\rho$ and magnetization channels $\phi$ to Gaussian order, an expansion of the action to second order in $g$ leads to

$$
\begin{aligned}
\mathcal{Z} & =\exp \left[-\int g\left(m^{2}-\rho^{2}\right)+\operatorname{Tr} \ln \hat{G}^{-1}\right. \\
& \left.-\frac{g^{2}}{2} \operatorname{Tr}\left(\hat{\Pi}_{+-} \hat{\Pi}_{-+}-\hat{\Pi}_{++} \hat{\Pi}_{--}\right)\right],
\end{aligned}
$$

where we have defined the spin-dependent polarization operator $\hat{\Pi}_{s s^{\prime}}=\hat{G}_{s} \hat{G}_{s^{\prime}}$, and $\hat{G}_{ \pm}^{-1}=\partial_{\tau}+\epsilon_{\hat{\mathbf{k}}}-\mu_{ \pm}+g \rho_{0} \mp$ $g|\mathbf{m}|$. The contact interaction means that an unphysical ultraviolet divergence arises from the term in the action that is second order in $g$. To remove it we must affect the standard regularization of the linear term $g\left(m^{2}-\rho^{2}\right)$, setting $g \mapsto \sqrt{2 / \pi} a / b-2(\sqrt{2 / \pi} a / b)^{2} A^{-1} \sum_{\mathbf{k}_{3,4}}^{\prime}\left(\epsilon_{\mathbf{k}_{1}}+\right.$ $\left.\epsilon_{\mathbf{k}_{2}}-\epsilon_{\mathbf{k}_{3}}-\epsilon_{\mathbf{k}_{4}}\right)^{-1}$ [19], where the prime indicates that the summation is subject to the momentum conservation condition $\mathbf{k}_{1}+\mathbf{k}_{2}=\mathbf{k}_{3}+\mathbf{k}_{4}$, and $A$ denotes the total area of one stacked layer.

Finally, after carrying out the remaining Matsubara summations, one obtains the following expression for the free energy:

$$
\begin{aligned}
& F=\sum_{\mathbf{k}, s= \pm} \epsilon_{\mathbf{k}}^{s} n_{s}\left(\epsilon_{\mathbf{k}}\right)+\sqrt{\frac{2}{\pi}} \frac{a}{b A} N_{+} N_{-} \\
& -2\left(\sqrt{\frac{2}{\pi}} \frac{a}{b A}\right)_{\mathbf{k}_{1,2,3,4}}^{2} \sum^{\prime} \frac{n_{+}\left(\epsilon_{\mathbf{k}_{1}}\right) n_{-}\left(\epsilon_{\mathbf{k}_{2}}\right)\left[n_{+}\left(\epsilon_{\mathbf{k}_{3}}\right)+n_{-}\left(\epsilon_{\mathbf{k}_{4}}\right)\right]}{\epsilon_{\mathbf{k}_{1}}+\epsilon_{\mathbf{k}_{2}}-\epsilon_{\mathbf{k}_{3}}-\epsilon_{\mathbf{k}_{4}}}
\end{aligned}
$$

where $n_{s}(\epsilon)=1 /\left[1+\mathrm{e}^{\beta\left(\epsilon-\mu_{s}-s|\mathbf{m}| \sqrt{2 / \pi} a / b\right)}\right]$ is the Fermi distribution, and $N_{s}=\sum_{\mathbf{k}} n_{s}\left(\epsilon_{\mathbf{k}}\right)$. To evaluate the final nine-dimensional integral in Eq. (4) numerically we employ the re-parameterization outlined in App. Ato reduce it to a four-dimensional integral. Moreover, as we are interested in searching for extrema in the free energy with changing polarization we can differentiate our expression with respect to magnetization, which at zero temperature further reduces the integral to just three dimensions.

To highlight the potential importance of fluctuation corrections we briefly study the contribution to the energy from particle-hole excitations around momentum $2 k_{\mathrm{F}}$. At zero temperature a non-analytic contribution to the free energy of the form $|m|^{3} \ln m^{2}$ emerges. The same non-analyticity was found diagrammatically in Refs. [3]. The formation of a finite magnetization increases the phase-space available for the formation of virtual intermediate pairs of particle-hole pairs, and this phase space enhancement donates a non-analytic term to the free energy giving the transition the potential for first order character. In the next section we study the effect that this non-analyticity has on the phase diagram.

\section{PHASE BEHAVIOR}

With the formal development of the theory complete we will now apply the formalism to explore the implications of ferromagnetism in the two-dimensional atomic 


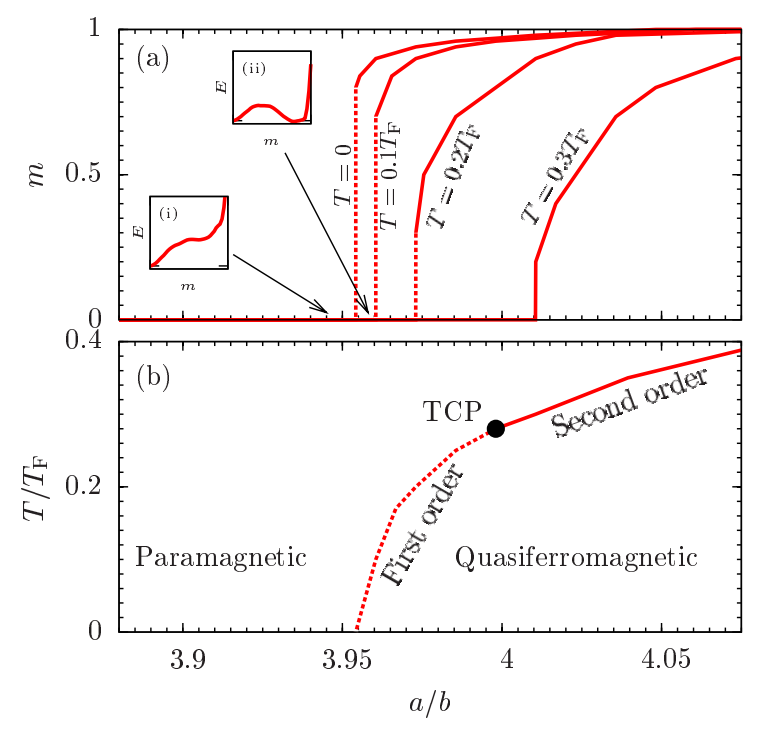

FIG. 1: (Color online) (a) The growth of magnetization $m$ with scattering length for different temperatures. The inset figures show the energy landscape with magnetization for $T=0$ either side of the first order transition. (b) the phase diagram of temperature with scattering length shows the first order (dashed red line) and second order (solid red line) (quasi)ferromagnetic ordering from the paramagnetic phase.

Fermi gas, and critically compare the results with the three-dimensional case [7]. Before we study the phase diagram of the fluctuation corrected free energy, to make contact with the conventional Stoner theory we first consider the result of a direct saddle point approximation scheme in which the second order term in the free energy is neglected. In this approximation at zero temperature the free energy is $F=\left(1+a / b \sqrt{2} \pi^{3 / 2}\right) \mu^{2} / 2 \pi+$ $\left(1-a / b \sqrt{2} \pi^{3 / 2}\right) m^{2} / 2 \pi \mu^{2}$. This expression is exact, and with magnetization featuring only as the lowest available term in a Landau expansion its analysis is straightforward. For $a<\sqrt{2} \pi^{3 / 2} b \approx 7.874 b$ this model predicts that the gas is paramagnetic, whereas for $a>\sqrt{2} \pi^{3 / 2} b$ the system is fully polarized, a scenario that remains unaltered with the introduction of population imbalance. An immediate corollary is that the spontaneous magnetization formed is independent of the local density, which also holds true when fluctuation corrections are taken into account. Therefore, within a trap, the entire atomic gas adopts the same polarization.

Having studied the mean-field limit, we now consider the repercussions of fluctuation corrections on the behavior of the magnetization. To orient our discussion, we first consider a gas with equal populations of up and down-spin atoms. As shown in Fig. 1(ai) at scattering lengths below $a \approx 3.945 b$ the energy profile possesses a single minimum at zero magnetization. With rising interaction strength a second minimum in the energy landscape develops at $m \approx 0.6$, which, with rising scattering length, deepens in Fig. 1(aii) to become the global minimum at $a \approx 3.953 b$ and $m \approx 0.8$. At

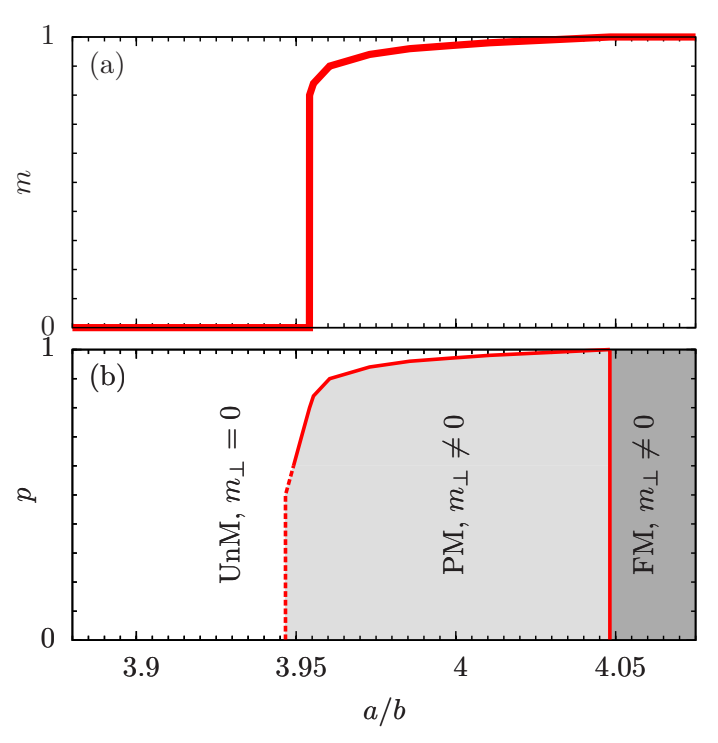

FIG. 2: (Color online) (a) The growth of magnetization $m$ with scattering length at $T=0$. (b) the $T=0$ phase diagram for imposed population imbalance $p$ with scattering length $a / b$ shows the first order (dashed red line) and second order (solid red line) ferromagnetic ordering from the unmagnetized (UnM) to the partially magnetized (PM) and fully magnetized (FM) regions, here unmagnetized refers to having no in-plane magnetization.

this scattering length the system undergoes a first order transition from $m=0$ into the polarized regime with $m \approx 0.8$. As shown in Fig. 1(a) with a further increase in the interaction strength the magnetization saturates at a scattering length $a \approx 4.048 b$. Fluctuation corrections have had significant impact: they have driven the ferromagnetic transition to a significantly weaker interaction strength $(a \approx 3.953 b)$ compared to the mean-field case $(a \approx 7.874 b)$. At this weaker interaction strength the $m^{2}$ term in the free energy has a positive coefficient, and the ordering is driven by the non-analytic $|m|^{3} \ln m^{2}$ term. The abetment of the transition by fluctuation corrections and reduction in interaction strength at which ferromagnetism is seen is common to both the two and three-dimensional cases, though in two dimensions the transition is immediately to full polarization at meanfield level and fluctuation corrections drive a first order transition at a weaker interaction strength.

We now turn to address the behavior of the phase transition at finite temperature in Fig. 1(a). Increasing temperature dulls the fluctuation corrections and the scattering length of the first order transition rises and the magnetization following the transition is reduced. Fig. 1(b) shows that at $T \approx 0.28 T_{\mathrm{F}}$ a tricritical point emerges and the system reverts to second order behavior. The Mermin-Wagner-Hohenberg theorem [20] states that although an ordered phase can exist in two dimensions at zero temperature, at any finite temperature fluctuations will destroy long range correlations in the system, and the state will be characterized by exponentially decaying 
correlation functions. Therefore we denote the ferromagnetic state as a "quasiferromagnet" (ferromagnet with fluctuating polarization direction). However, since the two-dimensional gas is experimentally realized in a series of disks, each one can couple to its neighbors and tunneling should stabilize the phase [12, 24]. Furthermore, the Mermin-Wagner-Hohenberg theorem is valid only in the thermodynamic limit and does not apply to finite-sized systems. For a two-dimensional Bose gas with attractive interactions it has been shown that a potential trap restricts the system and stabilizes a quasi-Bose Einstein condensate [21]. In a similar way the harmonic trap should stabilize a ferromagnetic phase. So far we have focused on how equilibrium properties can stabilize the ferromagnetic phase, however, there are also nonequilibrium aspects to consider. Within the current experimental realization of cold atom gas ferromagnetism three-body losses necessitate that the experiment be performed out of equilibrium. Following a quench small ferromagnetic domains are formed [22] which then grow steadily [10]. The final size of these ferromagnetic domains $\sim 6 / k_{\mathrm{F}}[10]$ at $T=0.1 T_{\mathrm{F}}$ and $k_{\mathrm{F}} a=2$ is small compared to the length-scale of the thermal fluctuations given by $a \exp \left[2 \pi\left(2 k_{\mathrm{F}} a / \pi-1\right) T_{\mathrm{F}} / T\right] \approx 10^{7} / k_{\mathrm{F}}[25]$. This means that at sufficiently low temperature fluctuations will not disrupt the ferromagnetic state and so in experiments a true ferromagnetic phase should be observed as shown in Fig. 1.

Having addressed the situation without population imbalance, we now consider how a fixed spin population imbalance influences the phase diagram. The two constituent species cannot interconvert so an initial population imbalance is maintained by the difference in their chemical potentials. However, if energetically favorable, the gas can become more polarized either by phase separation or the development of an in-plane magnetic moment. As shown in Fig. 1(ai and aii), at weak interactions such that $a \lesssim 3.945 b$, the energy monotonically increases with magnetization, but the magnetization remains pinned to the minimum value defined by the population imbalance $p$. However, with rising interaction strength a second minimum develops in the free energy landscape from $a \approx 3.945 b$ and $m \approx 0.6$. If that magnetization exceeds the population imbalance, then as shown in Fig. 2(b) the system will phase separate between this minimum and that at zero magnetization, with relative fractions governed by the Maxwell construction. When the emerging minimum becomes the global minimum at $m \approx 0.8$, then gases with a lower population imbalance enter this global minimum with an appropriate in-plane magnetic moment. As the magnetization of the minimum rises it envelops systems with higher population imbalance, and tracks the magnetization curve shown in Fig. 2(a) until it reaches full polarization at $a / b \approx 4.048$. Like the three-dimensional case, the population imbalance renders the characteristic interaction strength of the transition to be almost constant up to an imposed population imbalance of $p \approx 0.8$, which could be a key experimental signature of first order behavior.

\section{TRAPPED GEOMETRY}

Having addressed the phase behavior of a uniform system, to make contact with the experiment we now turn to address the atomic gas trapped within the spherical potential $V(\mathbf{r})=\omega r^{2} / 2$. Following the program developed in Refs. 10 12 we aim to minimize the free energy within the local density approximation using the kernel $f(\mathbf{r})=F(\mathbf{r})+V(\mathbf{r})\left[n_{+}(\mathbf{r})+n_{-}(\mathbf{r})\right]-\gamma_{+} n_{+}(\mathbf{r})+$ $\gamma_{-} n_{-}(\mathbf{r})$, here $F(\mathbf{r})$ denotes the energy kernel Eq. (4) evaluated with the local chemical potential at $\mathbf{r}$. The Lagrange multipliers $\gamma_{ \pm}$enforce the constraints of constant number of atoms imposed by the trap geometry $N_{\text {tot }}=\int\left[n_{+}(\mathbf{r})+n_{-}(\mathbf{r})\right] \mathrm{d}^{2} r$ and population imbalance $p \leq \int\left[n_{+}(\mathbf{r})-n_{-}(\mathbf{r})\right] \mathrm{d}^{2} r / N_{\text {tot }}$; without loss of generality we assume that $p \geq 0$ and therefore $\gamma_{+} \geq \gamma_{-}$. To study the effects of spatial density variations we invoke a local density approximation that enables the variational minimization $\delta f / \delta n_{s}(\mathbf{r})$ and yields the simultaneous equations for the effective local chemical potentials $\mu_{ \pm}(\mathbf{r})$ for the species in the rotated spin basis

$$
\begin{aligned}
& \mu_{ \pm}(\mathbf{r})=\gamma_{ \pm}-V(\mathbf{r})-\sqrt{\frac{2}{\pi}} \frac{a}{b A} n_{\mp}(\mathbf{r})+2\left[\sqrt{\frac{2}{\pi}} \frac{a}{b A}\right]_{\mathbf{k}_{1,2,3,4}}^{2} \sum_{n_{\mp}}^{\prime}\left(\epsilon_{\mathbf{k}_{2}}\right) \\
& \times \frac{n_{ \pm}\left(\epsilon_{\mathbf{k}_{1}}\right) \delta\left(\epsilon_{\mathbf{k}_{3}}-\mu_{ \pm}\right)+\left[n_{ \pm}\left(\epsilon_{\mathbf{k}_{3}}\right)+n_{\mp}\left(\epsilon_{\mathbf{k}_{4}}\right)\right] \delta\left(\epsilon_{\mathbf{k}_{1}}-\mu_{ \pm}\right)}{\epsilon_{\mathbf{k}_{1}}+\epsilon_{\mathbf{k}_{2}}-\epsilon_{\mathbf{k}_{3}}-\epsilon_{\mathbf{k}_{4}}} .
\end{aligned}
$$

These equations can be understood as having been constructed out of three orders of perturbation theory. The lowest, independent of the scattering length $a$, corresponds to the Thomas-Fermi approximation within the confining potential, the term first order in $a$ introduces the mean-field energy penalty of the interaction whereas the second order term introduces the energy associated with magnetic quantum fluctuations. The detailed study of the uniform system revealed that the polarization depends only on the interaction strength and not spatial density variations, meaning that the ratio $n_{+}(\mathbf{r}) / n_{-}(\mathbf{r})$ and therefore $\mu_{+}(\mathbf{r}) / \mu_{-}(\mathbf{r})$ is constant across the trap. Therefore, the two equations reduce to just one that is solved by iteration.

\section{A. Heuristic observations}

We first address what can be determined about the behavior of the atomic gas heuristically before presenting the results of the full solution of Eq. (5). To develop our intuition we focus on perhaps the most physical quantity that can be measured by experiment, namely the cloud size. To start the analysis we consider the non-interacting limit $a=0$ where the system is unpolarized and the effective chemical potentials given by Eq. (5) follow the familiar Thomas-Fermi form. The root 
mean square (RMS) radius would increase with population imbalance as $\left[1+\left(\frac{1-p}{1+p}\right)^{3 / 2}\right]^{1 / 2} / \sqrt{2}$ due to the increasing Fermi degeneracy pressure. With weak interactions $a \ll \sqrt{2} \pi^{3 / 2} b$ we need consider Eq. (5) only to first order in $a$ which yields $\mu_{ \pm}(\mathbf{r})=\gamma_{ \pm}-V(\mathbf{r})-a \max \left[\gamma_{\mp}-\right.$ $V(\mathbf{r}), 0] / 2^{1 / 2} \pi^{3 / 2} b$. The first order term reduces the effective chemical potential so to conserve the total number of trapped atoms we renormalize the Lagrange multipliers upwards from the Thomas Fermi value by a factor of $1+a(1-p) /(2 \pi)^{3 / 2} b(1+p)$ for the majority spin species and $1+a\left[2-\left(\frac{1-p}{1+p}\right)^{1 / 2}\right] /(2 \pi)^{3 / 2} b$ for the minority spin species. This reduction in the effective chemical potential and corresponding fall in local density can be understood in terms of an increase in the local pressure within the cloud due to the repulsive interactions between the atoms. This pressure inflates the cloud causing the RMS radius to rise through a factor of $1+a(1-p)(3 \sqrt{1+p}-$ $\sqrt{1-p}) / 2^{5 / 2} \pi^{3 / 2} b\left[(1+p)^{3 / 2}+(1-p)^{3 / 2}\right]$. Having analyzed the weakly interacting regime it is natural to also examine the strongly interacting limit. Here the atomic gas is fully polarized so $\mu_{-}=0$ and $\mu_{+}(\mathbf{r})=\gamma_{+}-V(\mathbf{r})$, meaning that the system is firmly in the Thomas Fermi regime. We again require that the number of particles is conserved which sets the majority spin Lagrange multiplier to rescale by a factor of $2^{1 / 2}$ from its original value if there were no population imbalance. Consequentially the enhanced Fermi degeneracy pressure dilates the RMS radius of a cloud with zero population imbalance by a factor of $2^{1 / 4}$. The key limits of weak and strong interactions hold true whatever the true theory of ferromagnetism so provide two valuable handles for potential experiments.

\section{B. Exact analysis of trapped behavior}

Having completed the overview of the trapped behavior we now turn to consider the ramifications of fluctuation corrections and self-consistently solve Eq. (5) for the chemical potentials $\mu_{ \pm}$. We then integrate over the trap to extract the full behavior of the experimental observables, namely cloud size, kinetic energy, and threebody loss rate, which for the mean-field limit are shown in Fig. 3. The same calculation repeated for fluctuation corrections is shown in Fig. 4. A useful reference throughout will be the complementary analysis in three dimensions [10, 11]. The orthodox Stoner mean-field theory predicts that at the onset of ferromagnetic ordering the system immediately fully polarizes across the entire trap at $a / b=\sqrt{2} \pi^{3 / 2} \approx 7.874$, whereas fluctuation corrections allow the cloud to adopt partial polarization over the window of scattering lengths $3.953 \lesssim a / b \lesssim 4.048$. In twodimensions as the entire gas polarizes at the same interaction strength striking features emerge at these respective interaction strengths. Current experiments [9] can probe scattering lengths to $\sim 10 \%$ accuracy, therefore in current experiments the fluctuation corrected transition will also appear to immediately give complete polarization.

To develop our intuition we first examine the projected

\section{Mean-field}

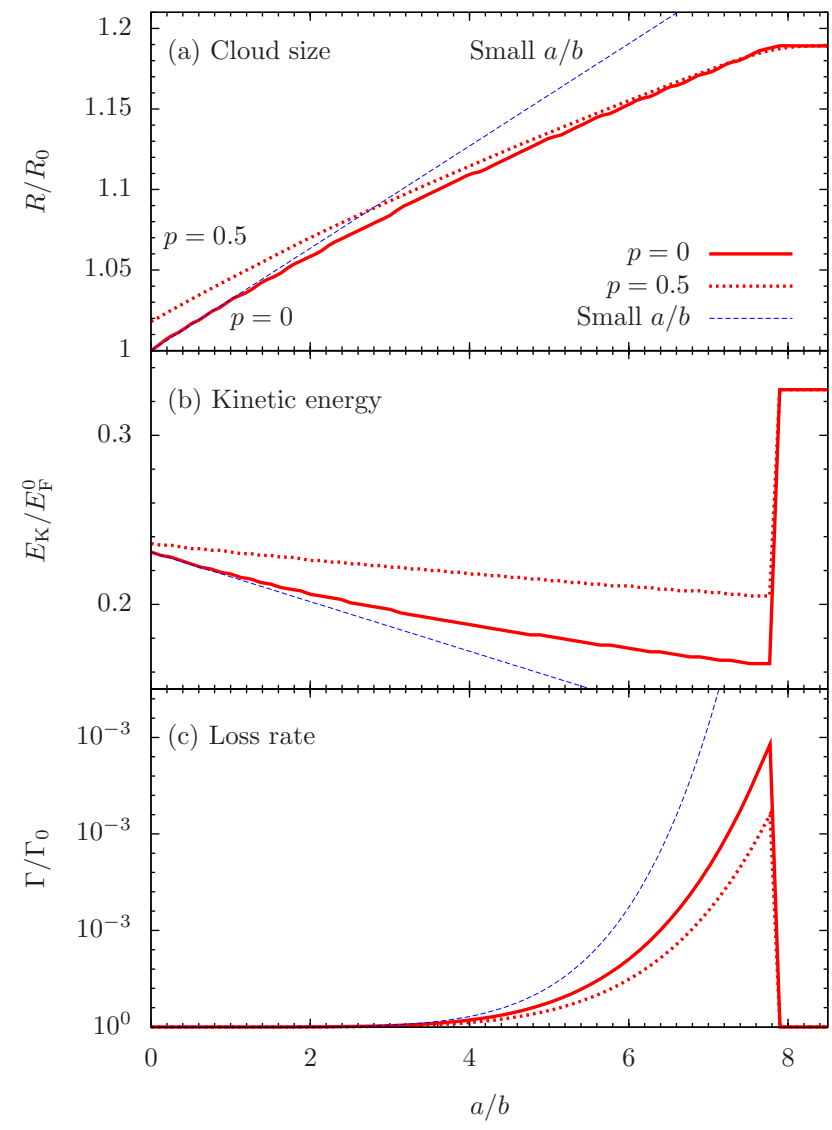

FIG. 3: (Color online) The variation of (a) cloud size, (b) kinetic energy, and (c) atom loss rate on ferromagnetic ordering with increasing scattering length $a / b$ for the orthodox Stoner mean-field theory case. The thin blue dashed line highlights the small $a / b$ behavior. The solid lines are at zero population imbalance whereas the dotted line is with an imposed population imbalance of 0.5 .

cloud size. In the mean-field limit (Fig. 3(a)) with weak interactions the RMS radius grows linearly with scattering length as the atoms repel each other within the trap. The radius grows following the universal scaling described above. Population imbalance causes the cloud to have an initially larger radius due to the increased Fermi degeneracy pressure. The entire cloud becomes fully polarized at the same scattering length, $a / b=\sqrt{2} \pi^{3 / 2}$, and at this point the cloud size immediately adopts its final inflated radius $R / R_{0}=2^{1 / 4}$, maintained by Fermi degeneracy pressure. This is in contrast to the threedimensional case [10, 11] in which the transition takes place over a range of interaction strengths, thus making the transition less distinct. Fig. 4(a) shows that fluctuation corrections drive the cloud expansion faster, causing it to dilate rapidly. In contrast to the three-dimensional case [10, 11], this pressure cannot drive the cloud to grow larger than the fully polarized size $2^{1 / 4} R_{0}^{\mathrm{RMS}}$. As the interaction strength is unaffected by the density of atoms, the transition occurs at the same scattering length 


\section{Fluctuation corrected}

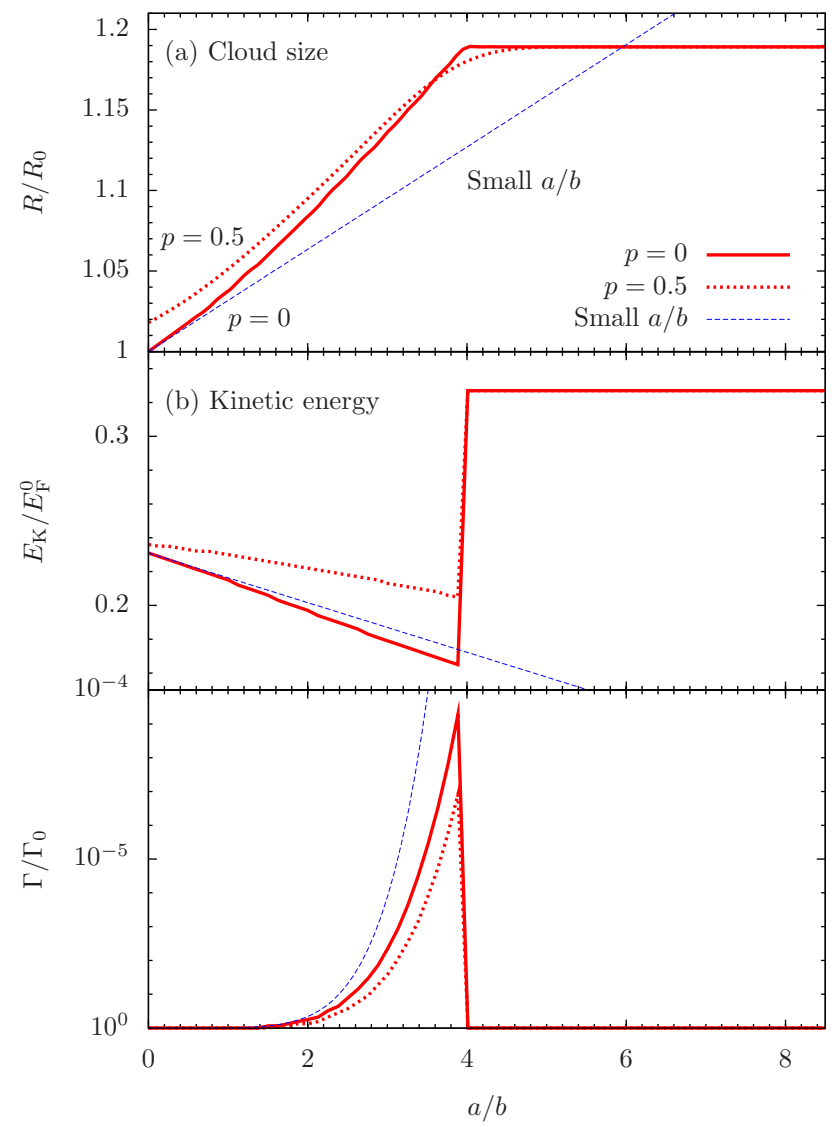

FIG. 4: (Color online) The variation of (a) cloud size, (b) kinetic energy, and (c) atom loss rate on ferromagnetic ordering with increasing scattering length $a / b$ when fluctuation corrections are taken into account. The thin blue dashed line highlights the small $a / b$ behavior. The solid lines are at zero population imbalance whereas the dotted line is with an imposed population imbalance of 0.5 .

\section{$a \approx 3.954 b$ seen in the uniform case.}

The total kinetic energy is probed experimentally by releasing the atoms from the trap and imaging them following a ballistic expansion. Starting from the meanfield analysis in Fig. 3(b), at zero interactions an initial population imbalance increases the kinetic energy due to the enlarged majority spin Fermi surface by a factor of $\left[1+\left(\frac{1-p}{1+p}\right)^{3 / 2}\right] / 2$. The weak interactions dilate the cloud, causing local density and kinetic energy to fall with the universal scaling $1-a(1-p)(3 \sqrt{1+p}+$ $\sqrt{1-p}) / 2^{5 / 2} \pi^{3 / 2} b\left[(1+p)^{3 / 2}+(1-p)^{3 / 2}\right]$. When the scattering length is increased beyond $a / b=\sqrt{2} \pi^{3 / 2}$ the entire gas becomes ferromagnetic and the atoms all enter the same Fermi surface. This Fermi surface is inflated and the kinetic energy plateaus at the final value that is $2^{1 / 2}$ times that for the non-interacting gas. When fluctuation corrections are taken into account one recovers the variation of kinetic energy shown in Fig. 4(b). The fluctuations drive the transition to take place at a reduced interaction strength $a \approx 3.954 b$ seen in the uniform case.

The atom loss rate due to three-body recombination is $\Gamma=\Gamma_{0}(a / b)^{6} \int n_{+}(\mathbf{r}) n_{-}(\mathbf{r})\left[n_{+}(\mathbf{r})+n_{-}(\mathbf{r})\right] \mathrm{d}^{2} r[26]$. In the recent experiment [9] the three-body loss was significant and forced the experiment to be performed rapidly and out of equilibrium, and here we study the situation in two-dimensions. We start by examining the meanfield limit in Fig. 3(c), which shows the three-body loss integrated over the entire trap. At weak interaction strengths the loss rate rises rapidly as $\Gamma=\Gamma_{0}(a / b)^{6} \mu^{4}(1-$ $\left.p^{2}\right) / 8 \pi^{2} \omega$. At a scattering length $a / b=\sqrt{2} \pi^{3 / 2}$ the gas across the entire trap becomes fully polarized so $n_{-}=0$ and therefore the three-body loss is completely cut off. This immediate elimination of loss contrasts the threedimensional case where loss remains until high interaction strengths, where it forces the experiment out of equilibrium [10], and also renormalizes the effective interaction strength 13]. Fig. 3 highlights how these effects are reduced in the two-dimensional case which could aid with the positive identification of the ferromagnetic phase. It can also be seen that population imbalance reduces atom loss primarily through reduction of the $n_{+}(\mathbf{r}) n_{-}(\mathbf{r})$ term. Having studied the mean-field limit we now look at the impact of fluctuation corrections on three-body loss in Fig. 4(c). The fluctuation corrections drive the ferromagnetic transition to take place at a reduced scattering length of $a / b=3.954$. This in turn means that the peak three-body loss $\left(\propto a_{\text {crit }}^{6}\right)$ is significantly reduced. This fall in loss rate will mean that an experiment searching for signatures of ferromagnetism can be performed nearer to the equilibrium regime which should yield clearer results.

\section{DISCUSSION}

In conclusion, on the repulsive side of the Feshbach resonance coupling of transverse magnetic fluctuations drives ferromagnetic ordering first order. We studied the specific variation of three experimental signatures of ferromagnetism: cloud size, release energy, and atom loss rate. The formalism highlighted the benefits of studying ferromagnetism in two rather than three dimensions. In two-dimensions the effective interaction strength is independent of density and therefore radius in the harmonic well. As the interaction strength is ramped upwards the entire gas will enter into the ferromagnetic phase at the same Feshbach field, whereas in three-dimensions the gas first enters the ferromagnetic state at the center. Therefore the signatures of the ferromagnetic phase are enhanced in two-dimensions, which should aid the exact characterization of the state. At weak interactions these observables displayed universal scaling, and the variation with an imposed population imbalance was also considered.

One intriguing possibility opened up by the new formalism developed to study fluctuation corrections is ferromagnetic reconstruction into a spin textured state, in a matter analogous to the FFLO state in superconductors. 
This has already been shown to be possible in three dimensions [8] and, with enhanced Fermi surface nesting in two dimensions, poses an interesting direction for future research.

The author thanks Ehud Altman, Andrew Green, Gyu-
Boong Jo, Wolfgang Ketterle, Ben Simons, and Joseph Thywissen for useful discussions. The author acknowledges the financial support of the Royal Commission for the Exhibition of 1851 and the Kreitman Foundation.
[1] E.C. Stoner, Proc. R. Soc. London 165, 372 (1957).

[2] J.A. Hertz, Phys. Rev. B 14, 1165 (1976); A.J. Millis, Phys. Rev. B 48, 7183 (1993); T. Moriya, Solid State Science 56 (Springer, Berlin, Heidelberg, 1985).

[3] D.L. Maslov, A.V. Chubukov and R. Saha, Phys. Rev. B 74, 220402(R) (2006). D.V. Efremov, J.J. Betouras and A. Chubukov, Phys. Rev. B 77, 220401(R) (2008).

[4] R.A. Duine and A.H. MacDonald. Phys. Rev. Lett. 95, 230403 (2005).

[5] For a review, see D. Belitz, T. Kirkpatrick, T. Vojta, Rev. Mod. Phys. 77, 579 (2005).

[6] A.A. Abrikosov and I.M. Khalatnikov, Soviet Phys. JETP 6, 888 (1958).

[7] G.J. Conduit and B.D. Simons, Phys. Rev. A 79, 053606 (2009)

[8] G.J. Conduit, A.G. Green and B.D. Simons. Phys. Rev. Lett. 103, 207201 (2009).

[9] G.-B. Jo, Y.-R. Lee, J.-H. Choi, C.A. Christensen, T.H. Kim, J.H. Thywissen, D.E. Pritchard and W. Ketterle, Science 325, 1521 (2009).

[10] G.J. Conduit and B.D. Simons. Phys. Rev. Lett. 103, 200403 (2009).

[11] L.J. LeBlanc, J.H. Thywissen, A.A. Burkov and A. Paramekanti, Phys. Rev. A 80, 013607 (2009).

[12] I. Berdnikov, P. Coleman and S.H. Simon, Phys. Rev. B 79224403 (2009).

[13] G.J. Conduit and E. Altman. arXiv:0911.2839

[14] R.A. Duine, M. Polini, H.T.C. Stoof and G. Vignale, Phys. Rev. Lett. 104, 220403 (2010).

[15] T. Chatterji, Journal of Alloys and Compounds 326, 15 (2001).

[16] Y.J. Chang, C.H. Kim, S.H. Phark, Y.S. Kim, J. Yu and T.W. Noh, Phys. Rev. Lett. 103, 057201 (2009).

[17] H. v. Löhneysen et al., Rev. Mod. Phys. 79, 1015 (2007).

[18] S. Pilati et al., arXiv:1004.1169v1; S.-Y. Chang, M. Randeria, and N. Trivedi, arXiv:1004.2680 1 .

[19] R.K. Pathria, Statistical Mechanics, Pergamon Press (1996).

[20] N.D. Mermin and H. Wagner, Phys. Rev. Lett. 22, 1133 (1966); P.C. Hohenberg, Phys. Rev. 158, 383 (1967).

[21] J.O. Andersen, U. Al Khawaja and H.T.C. Stoof, Phys. Rev. Lett. 88, 070407 (2002); U. Al Khawaja, J.O. Andersen, N.P. Proukakis and H.T.C. Stoof, Phys. Rev. A 66, 013615 (2002); C. Gies, B.P. van Zyl, S.A. Morgan and D.A.W. Hutchinson, Phys. Rev. A 69, 023616 (2004).

[22] M. Babadi, D. Pekker, R. Sensarma, A. Georges and E. Demler, arXiv:0908.3483.

[23] R.K. Bhaduri, S.M. Reimann, S. Viefers, A. Ghose Choudhury and M.K. Srivastava, J. Phys. B 33, 3895 (2000).

[24] D.S. Petrov, M. Holzmann and G.V. Shlyapnikov, Phys. Rev. Lett. 84, 2551 (2000).

[25] A. Auerbach, Interacting Electrons and Quantum Mag- netism, Springer (1994).

[26] D.S. Petrov, Phys. Rev. A 67, 010703(R) (2003).

[27] W. Zwerger, Science 325, 1507 (2009). 


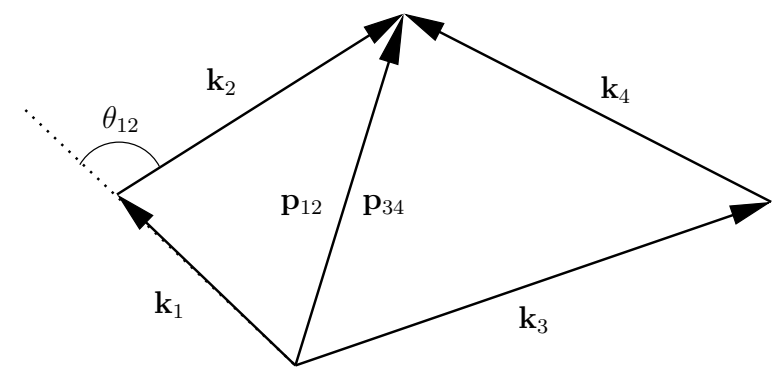

FIG. 5: The re-parameterization of the momenta $\mathbf{k}_{1,2,3,4} \cdot \theta_{12}$ represents the angle between $\mathbf{k}_{1}$ and $\mathbf{k}_{2}$. The two momenta $\mathbf{p}_{12}=\mathbf{k}_{1}+\mathbf{k}_{2}$ and $\mathbf{p}_{34}=\mathbf{k}_{3}+\mathbf{k}_{4}$ are constrained to be equal, $\mathbf{p}_{12}=\mathbf{p}_{34}$, by the Dirac delta function in Eq. A1.

\section{Appendix A: Computing the momentum space integral}

An important integral Eq. (4) encountered in this paper has the form

$$
\iiint \int \mathrm{d} \mathbf{k}_{1} \mathrm{~d} \mathbf{k}_{2} \mathrm{~d} \mathbf{k}_{3} \mathrm{~d} \mathbf{k}_{4} F\left(k_{1}, k_{2}, k_{3}, k_{4}\right) \delta\left(\mathbf{k}_{1}+\mathbf{k}_{2}-\mathbf{k}_{3}-\mathbf{k}_{4}\right) .
$$

To evaluate this integral one could substitute $\mathbf{k}_{4}=$ $\mathbf{k}_{1}+\mathbf{k}_{2}-\mathbf{k}_{3}$, and then integrate over the three parameters representing the lengths of vectors $\mathbf{k}_{1}, \mathbf{k}_{2}$, and $\mathbf{k}_{3}$, and a minimum of three relative angles between these vectors, giving a total of six integration parameters. However, since numerical integration generally becomes prohibitive with increasing number of dimensions we outline a scheme that takes advantage of the fact that the function $F$ depends only on the magnitude of the momenta to perform the angular integrals and leave a numerical integral over just the four vector lengths. A similar scheme has been developed in the three-dimensional case [7].

The integral is re-parameterized according to Fig. 5 The angular integral associated with vectors $\mathbf{k}_{1}$ and $\mathbf{k}_{2}$ is $\int_{0}^{2 \pi} 2 \pi k_{1} k_{2} \mathrm{~d} \theta_{12}$, where $\theta_{12}$ is the angle between $\mathbf{k}_{1}$ and $\mathbf{k}_{2}$. We now change the variable of the angular integral over $\theta_{12}$ to the vector $\mathbf{p}_{12}=\mathbf{k}_{1}+\mathbf{k}_{2}$ through the relationship $\cos \theta_{12}=\left(k_{1}^{2}+k_{2}^{2}-p_{12}^{2}\right) / 2 k_{1} k_{2}$ and so $\int_{0}^{2 \pi} \mathrm{d} \theta_{12}=$ $\int_{\left|k_{1}-k_{2}\right|}^{k_{1}+k_{2}} \mathrm{~d} p_{12} 8 \pi k_{1} k_{2} p_{12}\left[4 k_{1}^{2} k_{2}^{2}-\left(k_{1}^{2}+k_{2}^{2}-p_{12}^{2}\right)^{2}\right]^{-1 / 2}$. This expression, and an analogous one in $\mathbf{p}_{34}=\mathbf{k}_{3}+\mathbf{k}_{4}$, allows us to rewrite the original integral Eq. (A1) in terms of the parameters $p_{12}$ and $p_{34}$. The momentum conservation requirement is imposed by $\delta\left(\mathbf{k}_{1}+\mathbf{k}_{2}-\mathbf{k}_{3}-\mathbf{k}_{4}\right)$ which now introduces a new conservation law $\delta\left(\mathbf{p}_{12}-\mathbf{p}_{34}\right)$. This sets the two integration parameters equal, $p_{12}=p_{34}=p$, so there is just one integral over parameter $p$ remaining, and since the delta function constrains the angle between $\mathbf{p}_{12}$ and $\mathbf{p}_{34}$ we must also divide by the phase space associated with the angular integration of $2 \pi p$. We then obtain

$$
\begin{aligned}
& 32 \pi \iiint \int \mathrm{d} k_{1} \mathrm{~d} k_{2} \mathrm{~d} k_{3} \mathrm{~d} k_{4} \int_{\max \left(\left|k_{1}-k_{2}\right|,\left|k_{3}-k_{4}\right|\right)}^{\min \left(k_{1}+k_{2}, k_{3}+k_{4}\right)} \mathrm{d} p \times \\
& \frac{F\left(k_{1}, k_{2}, k_{3}, k_{4}\right) k_{1} k_{2} k_{3} k_{4} p}{\sqrt{4 k_{1}^{2} k_{2}^{2}-\left(k_{1}^{2}+k_{2}^{2}-p^{2}\right)^{2}} \sqrt{4 k_{3}^{2} k_{4}^{2}-\left(k_{3}^{2}+k_{4}^{2}-p^{2}\right)^{2}}} .
\end{aligned}
$$

Finally, we note that the integral over variable $p$ is Carlson's standard elliptic integral of the first kind, which we denote by $R_{\mathrm{F}}$. This yields the final result

$$
\begin{aligned}
& 32 \pi \iiint \int \mathrm{d} k_{1} \mathrm{~d} k_{2} \mathrm{~d} k_{3} \mathrm{~d} k_{4} F\left(k_{1}, k_{2}, k_{3}, k_{4}\right) \times \\
& \Theta\left(k_{1}+k_{2}-\left|k_{3}-k_{4}\right|\right) \Theta\left(k_{3}+k_{4}-\left|k_{1}-k_{2}\right|\right) \times \\
& \frac{R_{\mathrm{F}}\left(0,1+\left|\frac{\left[\left(k_{1}+k_{2}\right)^{2}-\left(k_{3}-k_{4}\right)^{2}\right]\left[\left(k_{1}-k_{2}\right)^{2}-\left(k_{3}+k_{4}\right)^{2}\right]}{\left[\left(k_{1}+k_{2}\right)^{2}-\left(k_{3}+k_{4}\right)^{2}\right]\left[\left(k_{1}-k_{2}\right)^{2}-\left(k_{3}-k_{4}\right)^{2}\right]}\right|, 1\right)}{\sqrt{\left|\left[\left(k_{1}-k_{2}\right)^{2}-\left(k_{3}-k_{4}\right)^{2}\right]\left[\left(k_{1}+k_{2}\right)^{2}-\left(k_{3}+k_{4}\right)^{2}\right]\right|}} .
\end{aligned}
$$

The term introduced to compensate for the angular integrals can be efficiently computed by a suitable numerical library. This four-dimensional integral is now better suited to computational evaluation than the sixdimensional form of the original expression Eq. (A1). 